\title{
On Two-Dimensional Navier-Stokes Flows with Rotational Symmetries
}

\author{
By \\ Cheng He and Tetsuro Miyakawa \\ (Academia Sinica, China, and Kanazawa University, Japan)
}

\begin{abstract}
Navier-Stokes flows are found on $\boldsymbol{R}^{2}$ that decay in time more rapidly than observed in general. The decay rate is determined in accordance with the order of symmetry with respect to the action of dihedral groups of orthogonal transformations. Contrary to the previous work [13], the basic existence result is proved with no restriction on the size of initial data. Our result extends that of [3] under different assumptions on the initial data. Unlike [3], the proofs are all carried out without using estimates for the associated vorticity transport equations.

Key Words and Phrases. Navier-Stokes system, Cauchy problem, Group symmetry, Asymptotic behavior, Weighted estimate.

2000 Mathematics Subject Classification Numbers. 35Q30, 76D05.
\end{abstract}

\section{Statement of the results}

This paper studies the Cauchy problem for the Navier-Stokes system in $\boldsymbol{R}^{2}$ :

$$
\begin{array}{ll}
\partial_{t} u+u \cdot \nabla u=\Delta u-\nabla p, & \left(x \in \boldsymbol{R}^{2}, t>0\right), \\
\nabla \cdot u=0, & \left(x \in \boldsymbol{R}^{2}, t \geq 0\right), \\
\lim _{|x| \rightarrow \infty} u=0,\left.\quad u\right|_{t=0}=a . &
\end{array}
$$

Here, $\quad x=\left(x_{1}, x_{2}\right) \in \boldsymbol{R}^{2}, \quad t \geq 0, \quad \partial_{t}=\partial / \partial t ; \quad u=u(x, t)=\left(u_{k}(x, t)\right)_{k=1}^{2}$ and $p=$ $p(x, t)$ denote unknown velocity and pressure, respectively; and $a=a(x)$ is a given initial velocity. The kinematic viscosity is normalized. The standard notation is used for differential operators and function spaces and the summation convention is employed for repeated indices.

We are interested in finding a (unique) solution $u$ to (1.1) that satisfies

$$
\|u(t)\|_{r} \leq c(1+t)^{-(m+1) / 2-(1-1 / r)} \quad(1 \leq r \leq \infty)
$$

for some $m \in N \cup\{0\}$. (Here and in what follows $\|\cdot\|_{r}$ denotes the $L^{r}$-norm.) In view of the structure of solutions to parabolic equations, it is reasonable to guess that such solutions would decay sufficiently rapidly as $t+|x| \rightarrow \infty$; and our purpose is to specify a class of initial data that provide us with solutions satisfying (1.2). 
To be more precise, let us introduce some notation. Consider the two function spaces

$$
\begin{aligned}
& \boldsymbol{L}_{\sigma}^{r}=\left\{v \in \boldsymbol{L}^{r}\left(\boldsymbol{R}^{2}\right): \nabla \cdot v=0\right\}, \\
& \boldsymbol{L}_{\pi}^{r}=\left\{v=\nabla p \in \boldsymbol{L}^{r}\left(\boldsymbol{R}^{2}\right): p \in L_{\mathrm{loc}}^{r}\left(\boldsymbol{R}^{2}\right)\right\},
\end{aligned}
$$

Then the following Helmholtz decomposition holds:

$$
\boldsymbol{L}^{r}\left(\boldsymbol{R}^{2}\right)=\boldsymbol{L}_{\sigma}^{r} \oplus \boldsymbol{L}_{\pi}^{r} ; \quad\left(\boldsymbol{L}_{\sigma}^{r}\right)^{*}=\boldsymbol{L}_{\sigma}^{r^{\prime}}, \quad\left(\boldsymbol{L}_{\sigma}^{r}\right)^{\perp}=\boldsymbol{L}_{\pi}^{r^{\prime}} \quad\left(r^{\prime}=r /(r-1)\right) .
$$

Here and in what follows $\boldsymbol{L}^{r}$ will stand for $L^{r}$-spaces of vector or tensor-valued functions. The associated bounded projection $P: \boldsymbol{L}^{r}\left(\boldsymbol{R}^{2}\right) \rightarrow \boldsymbol{L}_{\sigma}^{r}$ is written as

$$
(P f)_{j}=P_{j k} f_{k}, \quad \widehat{P}_{j k} f_{k}(\xi)=\left(\delta_{j k}-\xi_{j} \xi_{k} /|\xi|^{2}\right) \hat{f}_{k}(\xi), \quad j=1,2,
$$

by using the Fourier transform. Since $u \cdot \nabla u=\partial_{j}\left(u_{j} u\right)=\nabla \cdot(u \otimes u)$ because $\nabla \cdot u=0$, we have $P(u \cdot \nabla u)=P \nabla \cdot(u \otimes u)=\nabla \cdot P(u \otimes u)$; so problem (1.1) is written in the form of the integral equation

$$
\begin{aligned}
u(t) & =E_{t} * a-\int_{0}^{t} \nabla E_{t-\tau} *[P(u \otimes u)](\tau) d \tau \\
& =E_{t} * a-\int_{0}^{t}(\nabla V)(t-\tau) *(u \otimes u)(\tau) d \tau \\
& \equiv\left(\int E_{t}(x-y) a_{j}(y) d y-\int_{0}^{t} \int\left(\partial_{\ell} V_{j k}\right)(x-y, t-\tau)\left(u_{\ell} u_{k}\right)(y, \tau) d y d \tau\right)_{j=1}^{2},
\end{aligned}
$$

where $E_{t}(x)=(4 \pi t)^{-1} \exp \left(-|x|^{2} / 4 t\right)$, and $V$ is the kernel function of $E_{t} * P$ :

$$
V(x, t)=\left(V_{j k}(x, t)\right)_{j, k=1}^{2}, \quad V_{j k}(x, t)=E_{t}(x) \delta_{j k}+\int_{0}^{\infty} \partial_{j} \partial_{k} E_{t+\tau}(x) d \tau .
$$

Given $a \in \boldsymbol{L}_{\sigma}^{2}$, we call $u:[0, \infty) \rightarrow \boldsymbol{L}_{\sigma}^{2}$ a weak solution to problem (1.1) with initial value $a$, if $u \in L^{\infty}\left(0, \infty: \boldsymbol{L}_{\sigma}^{2}\right), \nabla u \in L^{2}\left(0, \infty: \boldsymbol{L}^{2}\right)$, and

$$
-\int_{0}^{\infty}\left\langle u, \partial_{t} \phi\right\rangle d t+\int_{0}^{\infty}\langle\nabla u, \nabla \phi\rangle d t+\int_{0}^{\infty}\langle u \cdot \nabla u, \phi\rangle d t=\langle a, \phi(\cdot, 0)\rangle
$$

for all $\phi \in \boldsymbol{C}_{c}^{\infty}\left(\boldsymbol{R}^{2} \times[0, \infty)\right)$ such that $\nabla_{x} \cdot \phi(x, t)=0$, where $\langle\cdot, \cdot\rangle$ denotes the duality pairing. In the case of two space dimensions, a global-in-time weak solution exists uniquely for all $a \in \boldsymbol{L}_{\sigma}^{2}$; and furthermore, every weak solution $u$ is a strong solution in the sense that $u$ satisfies the integral equation (1.3) for each $t>0$. For the basic properties of weak solutions, we refer to [11]. We here note only that every weak solution $u$ is smooth for $t>0$ and $x \in \boldsymbol{R}^{2}$ and satisfies the energy equality 


$$
\|u(t)\|_{2}^{2}+2 \int_{s}^{t}\|\nabla u\|_{2}^{2} d \tau=\|u(s)\|_{2}^{2} \quad \text { for all } t \geq s \geq 0 .
$$

Therefore, in this paper we do not distinguish between weak and strong solutions and merely say a solution to problem (1.1). Our first result is

Theorem 1.1. Let the initial velocity a satisfy $\nabla \cdot a=0$ and

$$
c_{0}=\sup (1+|y|)^{3}|a(y)|<\infty, \quad c_{1}=\int(1+|y|)|a(y)| d y<\infty .
$$

Then the solution $u$ of (1.1) satisfies

$$
|u(x, t)| \leq\left\{\begin{array}{l}
c(1+|x|)^{-3}, \\
c(1+t)^{-3 / 2},
\end{array} \quad\|u(t)\|_{r} \leq c(1+t)^{-1 / 2-(1-1 / r)} \quad(1 \leq r \leq \infty) .\right.
$$

Hereafter, integration with respect to the space variables will be performed over $\boldsymbol{R}^{2}$ unless otherwise specified. Theorem 1.1 was first proved in [13] under the additional assumption that $c_{0}+c_{1}$ be small; see also [2], [5] for the Cauchy problem, and [8], [10] for the exterior problem. Our assumption (1.5) requires no restriction on the size of $a$. However, we should note that the moment condition $\int(1+|y|)|a(y)| d y<\infty$ given in (1.5) is not preserved under the time evolution. Indeed, as shown in [2], we know that

$$
\int(u \otimes u)(y, t) d y=c(t) I \quad \text { provided that } \quad \int(1+|x|)|u(x, t)| d x \leq c .
$$

In this sense, our assumptions on $a$ given in Theorem 1.1 are stringent. We need the moment condition in (1.5) in our present situation in order to avoid the appearance of the factor $\log (1+|x|)$ in estimating $(1+|x|)^{3}|u(x, t)|$, so that we can deduce our conclusion in the form of (1.6). It is desirable to replace the moment condition by some other (less stringent) one.

By Theorem 1.1 there always exists a solution $u$ decaying like $t^{-1 / 2-(1-1 / r)}$ in $\boldsymbol{L}^{r}$, provided the initial velocity $a$ satisfies the required conditions. Note in particular that $\|u(t)\|_{2}=O\left(t^{-1}\right)$ as $t \rightarrow \infty$; so the result of [20] suggests that we would need some symmetry condition to find solutions which decay more rapidly than stated in Theorem 1.1. In fact, [16] proves

Theorem $1.2([16])$. Under the assumption of Theorem 1.1, the solution $u$ satisfies

$$
\|u(t)\|_{r} \geq c_{r} t^{-1 / 2-(1-1 / r)} \quad \text { for all } 1 \leq r \leq \infty \text { and large } t>0,
$$

if and only if

$$
\left(\int(y \otimes a) d y, \int_{0}^{\infty} \int(u \otimes u) d y d \tau\right) \neq(0, c I) \quad \text { for all } c \in \boldsymbol{R} .
$$


We know (see [2]) that if $(1+|x|) a \in \boldsymbol{L}^{1}\left(\boldsymbol{R}^{2}\right)$ and $\nabla \cdot a=0$, the matrix

$$
\int(y \otimes a) d y=\left(\int y_{j} a_{k}(y) d y\right)_{j, k=1}^{2}
$$

is anti-symmetric.

By Theorems 1.1 and 1.2, we can reasonably expect that some symmetry conditions would be needed for finding solutions $u$ such that $\|u(t)\|_{r}=$ $o\left(t^{-1 / 2-(1-1 / r)}\right)$ as $t \rightarrow \infty$. In this paper we specify, in terms of the groupinvariance property, a class of the initial data for which the corresponding solutions satisfy

$$
\left(\int(y \otimes a) d y, \int_{0}^{\infty} \int(u \otimes u) d y d \tau\right)=(0, c I) .
$$

By Theorems 1.1 and 1.2, condition (1.7) ensures that the solutions $u$ decay like $o\left(t^{-1 / 2-(1-1 / r)}\right)$ as $t \rightarrow \infty$.

To this end, we follow [3] and employ the dihedral groups $\mathscr{D}_{m}, m \in \boldsymbol{N}$, $m \geq 3$ ([7], [17]), of orthogonal transformations on $\boldsymbol{R}^{2}$. Consider the matrices

$$
A_{m}=\left(\begin{array}{cc}
\cos (2 \pi / m) & -\sin (2 \pi / m) \\
\sin (2 \pi / m) & \cos (2 \pi / m)
\end{array}\right), \quad J=\left(\begin{array}{cc}
1 & 0 \\
0 & -1
\end{array}\right) .
$$

The dihedral group $\mathscr{D}_{m}$ is defined to be the (finite) subgroup of $O(2)$ generated by $\left\{A_{m}, J\right\}$. We easily see that the finite group $\mathscr{D}_{m}$ has order $2 m$ and the cyclic group $\mathscr{C}_{m}$ of order $m$ generated by $A_{m}$ is a normal subgroup of $\mathscr{D}_{m}$.

Let $G \subset O(2)$. We say that a vector field $v$ (resp. a scalar field $p$ ) on $\boldsymbol{R}^{2}$ is covariant with the action of $G$, or simply $G$-covariant, if

$$
v(A x)=A v(x) \quad(\text { resp. } p(A x)=p(x)) \quad \text { for all } A \in G .
$$

Note that if $p$ is a $G$-covariant scalar field, then $\nabla p$ is a $G$-covariant vector field. Furthermore, if a vector field $v$ is $G$-covariant, then the associated vorticity $\omega=\nabla \times v=\partial_{1} v_{2}-\partial_{2} v_{1}$ is also $G$-covariant in the sense that

$$
\omega(A x)=(\operatorname{det} A) \omega(x) \quad \text { for all } A \in G .
$$

Now we can state our second main result in this paper.

Theorem 1.3. Let $m \in N$. Suppose that $a$ is $\mathscr{D}_{m+2}$-covariant and satisfies

$$
\sup (1+|y|)^{m+3}|a(y)|<\infty, \quad \int(1+|y|)^{m+1}|a(y)| d y<\infty .
$$

Then the corresponding solution $u(\cdot, t)$ is $\mathscr{D}_{m+2}$-covariant for each $t>0$ and satisfies 


$$
\begin{gathered}
|u(x, t)| \leq c(1+|x|)^{-(m+3)}, \quad|u(x, t)| \leq c(1+t)^{-(m+3) / 2}, \\
\|u(t)\|_{r} \leq c(1+t)^{-(m+1) / 2-(1-1 / r)} \quad(1 \leq r \leq \infty) .
\end{gathered}
$$

Here again, the moment condition in (1.11) is not preserved during the time evolution.

Brandolese [3] proves (1.12) and (1.13) for $r>2$ under different assumptions on the initial data. He first deduces some decay result for the associated vorticity and then applies the Biot-Savart law. We directly estimate velocity fields $u$ in (weighted) $L^{r}$-spaces, with the aid of the Taylor expansion of the kernel functions in (1.3), to prove (1.12) and (1.13) for $1 \leq r \leq \infty$. The use of the vorticity is restricted to the study of algebraic structures of the resulting Taylor polynomials. As a consequence, we had to adopt assumption (1.11) which does not involve smoothness of initial data, but requires a stringent moment condition. But, we hope our approach will be effective in studying Navier-Stokes flows with symmetries in a more general unbounded domain.

We shall prove Theorem 1.1 in Section 2, while Theorem 1.3 will be proved in Section 3. In proving Theorem 1.1, we first show that some well-known $L^{r}$-estimates hold uniformly for our approximate solutions to (1.3). These $L^{r}$ estimates are then applied to proving appropriate $L^{2}$ and $L^{\infty}$-estimates with weights. These weighted estimates, combined with the assumptions on the initial data, then provide Theorem 1.1 with no restriction on the size of initial data.

As will be seen from (2.1) and (2.2) below, our construction of approximate solutions differs from those given in the other works; namely, we define our iteration scheme, replacing $u \cdot \nabla u$ not with $u^{n} \cdot \nabla u^{n}$ (or $\bar{u}^{n} \cdot \nabla u^{n}$ with $\bar{u}$ some regularization of $u$ ), but with $u^{n-1} \cdot \nabla u^{n}$. We have adopted our scheme, not only because we need the energy conservation, but also because we have to deduce such pointwise bounds as $\left|u^{n}(x, t)\right| \leq c(1+|x|)^{-3}$, with $c$ independent of $n$, for the approximate solutions $\left\{u^{n}\right\}$. Our method enables us to deduce uniform bounds of this kind for $\left\{u^{n}\right\}$ by a simple induction argument. However, it seems impossible to invoke the compactness method to extract a subsequence from $\left\{u^{n}\right\}$ that is expected to tend to our desired solution; in other words, we have to prove that the whole sequence $\left\{u^{n}\right\}$ converges to the solution. This will be done at the end of Section 2 (see Lemma 2.4), by using the fact that the space-dimension is 2 .

\section{Proof of Theorem 1.1}

Problem (1.1) is written as

$$
\frac{d u}{d t}+A u=-P(u \cdot \nabla u)=-P \nabla \cdot(u \otimes u), \quad u(0)=a \in \boldsymbol{L}_{\sigma}^{r},
$$


where

$$
A=-\Delta \quad \text { with } D(A)=\boldsymbol{L}_{\sigma}^{r} \cap \boldsymbol{W}^{2, r}\left(\boldsymbol{R}^{2}\right) .
$$

In this paper we employ the approximate solutions $\left\{u^{n}\right\}_{n \geq 0}$ defined by

$$
\begin{aligned}
& \frac{d u^{n}}{d t}+A u^{n}=-P\left(u^{n-1} \cdot \nabla u^{n}\right)=-P \nabla \cdot\left(u^{n-1} \otimes u^{n}\right), \\
& u^{n}(0)=a, \quad u^{0}(t)=E_{t} * a .
\end{aligned}
$$

The corresponding integral equation is:

$$
\begin{aligned}
u^{n}(t) & =u^{0}(t)-\int_{0}^{t} E_{t-\tau} * P \nabla \cdot\left(u^{n-1} \otimes u^{n}\right)(\tau) d \tau \\
& =E_{t} * a-\int_{0}^{t}(\nabla V)(t-\tau) *\left(u^{n-1} \otimes u^{n}\right)(\tau) d \tau \\
& =E_{t / 2} * u^{n}(t / 2)-\int_{t / 2}^{t}(\nabla V)(t-\tau) *\left(u^{n-1} \otimes u^{n}\right)(\tau) d \tau
\end{aligned}
$$

This section proves Theorem 1.1. To do so, we first prepare various estimates for $u^{n}$ which are uniform in the parameter $n$. We begin with

Lemma 2.1. (i) Let $1 \leq q \leq 2$ and let $a \in \boldsymbol{L}_{\sigma}^{2} \cap \boldsymbol{L}^{q}\left(\boldsymbol{R}^{2}\right)$. Then we have

$$
\begin{aligned}
& \left\|u^{n}(t)\right\|_{r} \leq \begin{cases}c(1+t)^{-(1 / q-1 / r)} & (q \leq r \leq 2), \\
c t^{-(1 / q-1 / r)} & (2<r \leq \infty),\end{cases} \\
& \left\|\nabla u^{n}(t)\right\|_{r} \leq c t^{-1 / 2-(1 / q-1 / r)} \quad(q \leq r \leq \infty) .
\end{aligned}
$$

(ii) Let $a \in \boldsymbol{L}_{\sigma}^{2}$ and $(1+|x|) a \in \boldsymbol{L}^{1}\left(\boldsymbol{R}^{2}\right)$. Then

$$
\begin{gathered}
\left\|u^{n}(t)\right\|_{r} \leq \begin{cases}c(1+t)^{-1 / 2-(1-1 / r)} & (1 \leq r \leq 2), \\
c t^{-1 / 2-(1-1 / r)} & (2<r \leq \infty),\end{cases} \\
\left\|\nabla u^{n}(t)\right\|_{r} \leq c t^{-1-(1-1 / r)} \quad(1 \leq r \leq \infty) .
\end{gathered}
$$

In all of the above estimates, the constants $c>0$ are independent of $n$.

Proof. For simplicity in notation, we write $u=u^{n}$ and $v=u^{n-1}$. The standard energy method applied to (2.1) gives

$$
\|u(t)\|_{2}^{2}+2 \int_{s}^{t}\|\nabla u\|_{2}^{2} d \tau=\|u(s)\|_{2}^{2} \quad(0 \leq s \leq t) .
$$

Moreover, we know ([9], [20]) that if $a \in \boldsymbol{L}_{\sigma}^{2} \cap \boldsymbol{L}^{q}\left(\boldsymbol{R}^{2}\right), 1 \leq q \leq 2$, then

$$
u \in B C\left([0, \infty): \boldsymbol{L}^{r}\left(\boldsymbol{R}^{2}\right)\right), \quad\|u(t)\|_{r} \leq c_{q r}(1+t)^{-(1 / q-1 / r)}, \quad(q \leq r \leq 2),
$$


with $c=c_{q r}>0$ independent of $n$. When $a \in \boldsymbol{L}_{\sigma}^{2}$ and $(1+|x|) a \in \boldsymbol{L}^{1}\left(\boldsymbol{R}^{2}\right)$, we know that

$$
\|u(t)\|_{r} \leq c_{r}(1+t)^{-1 / 2-(1-1 / r)} \quad(1 \leq r \leq 2),
$$

with $c_{r}>0$ independent of $n$; see [14], [15].

We next multiply $(2.1)$ by $2 A u$, integrate by parts and apply the estimates:

$$
\|u\|_{4}^{2} \leq c\|u\|_{2}\|\nabla u\|_{2}, \quad\left\|\nabla^{2} u\right\|_{2} \leq c\|A u\|_{2}
$$

to get

$$
\begin{aligned}
\frac{d}{d t}\|\nabla u\|_{2}^{2}+2\|A u\|_{2}^{2} & =-2(v \cdot \nabla u, A u) \leq 2\|v\|_{4}\|\nabla u\|_{4}\|A u\|_{2} \\
& \leq c\|v\|_{2}^{1 / 2}\|\nabla v\|_{2}^{1 / 2}\|\nabla u\|_{2}^{1 / 2}\|A u\|_{2}^{3 / 2} \\
& \leq\|A u\|_{2}^{2}+c\|v\|_{2}^{2}\|\nabla v\|_{2}^{2}\|\nabla u\|_{2}^{2} .
\end{aligned}
$$

Since $v$ also satisfies (2.3), it follows that $\|v(t)\|_{2} \leq\|v(s)\|_{2}$ for $0 \leq s<t$; so we get

$$
\frac{d}{d t}\|\nabla u\|_{2}^{2}+\|A u\|_{2}^{2} \leq c\|v(s)\|_{2}^{2}\|\nabla v\|_{2}^{2}\|\nabla u\|_{2}^{2} \quad \text { whenever } 0 \leq s \leq t
$$

Hence

$$
\frac{d}{d t}\left((t-s)\|\nabla u\|_{2}^{2}\right)+(t-s)\|A u\|_{2}^{2} \leq\|\nabla u\|_{2}^{2}+c\|v(s)\|_{2}^{2}\|\nabla v\|_{2}^{2}\left((t-s)\|\nabla u\|_{2}^{2}\right)
$$

for $0 \leq s<t$. Integrating (2.6) from $s$ to $t$ and applying (2.3) gives

$$
\begin{aligned}
& (t-s)\|\nabla u(t)\|_{2}^{2}+\int_{s}^{t}(\tau-s)\|A u\|_{2}^{2} d \tau \\
& \quad \leq c\|u(s)\|_{2}^{2}+c\|v(s)\|_{2}^{2} \int_{s}^{t}\|\nabla v\|_{2}^{2}\left((\tau-s)\|\nabla u\|_{2}^{2}\right) d \tau .
\end{aligned}
$$

By Gronwall's lemma, we obtain

$$
\begin{aligned}
(t-s)\|\nabla u(t)\|_{2}^{2} & \leq c\|u(s)\|_{2}^{2} \exp \left(c\|v(s)\|_{2}^{2} \int_{s}^{t}\|\nabla v\|_{2}^{2} d \tau\right) \\
& \leq c\|u(s)\|_{2}^{2} \exp \left(c\|v(s)\|_{2}^{4}\right) .
\end{aligned}
$$

We insert (2.8) in the last integral of (2.7) and apply (2.3) for $v$, to deduce

$$
(t-s)\|\nabla u(t)\|_{2}^{2}+\int_{s}^{t}(\tau-s)\|A u\|_{2}^{2} d \tau \leq c\|u(s)\|_{2}^{2}\left(1+\|v(s)\|_{2}^{4} \exp \left(c\|v(s)\|_{2}^{4}\right)\right) .
$$


Proof of (i). Since $a \in \boldsymbol{L}^{q}\left(\boldsymbol{R}^{2}\right) \cap \boldsymbol{L}_{\sigma}^{2}$ by assumption, (2.4) shows $\|u(t)\|_{2}^{2} \leq$ $c(1+t)^{-(2 / q-1)}$. We set $s=t / 2$ in (2.9) and apply $\|v(s)\|_{2} \leq\|v(0)\|_{2}=\|a\|_{2}$, to get

$$
(t / 2)\|\nabla u(t)\|_{2}^{2} \leq c\|u(t / 2)\|_{2}^{2}\left(1+\|a\|_{2}^{4} \exp \left(c\|a\|_{2}^{4}\right)\right) \leq c(1+t)^{-(2 / q-1)},
$$

and so $\|\nabla u(t)\|_{2} \leq c t^{-1 / q}$. Since the space dimension is 2 , this gives

$$
\|u(t)\|_{\mathrm{BMO}} \leq c\|\nabla u(t)\|_{2} \leq c t^{-1 / q} \quad(1 \leq q \leq 2),
$$

with BMO the John-Nirenberg space of functions of bounded mean oscillation ([1], [19]).

Let $2<r<\infty$. From $\|f\|_{r} \leq c_{r}\|f\|_{\mathrm{BMO}}^{1-2 / r}\|f\|_{2}^{2 / r}$, we get

$$
\|u(t)\|_{r} \leq c t^{-(1 / q-1 / r)} \quad(2<r<\infty) .
$$

Choosing $q=2$ we have $\|v(t)\|_{4} \leq c t^{-1 / 4}$, and so

$$
\begin{aligned}
\|u(t)\|_{\infty} & \leq c t^{-1 / 2}\|u(t / 2)\|_{2}+c \int_{t / 2}^{t}(t-\tau)^{-3 / 4}\|v\|_{4}\|\nabla u\|_{2} d \tau \\
& \leq c t^{-1 / q}+c \int_{t / 2}^{t}(t-\tau)^{-3 / 4} \tau^{-(1 / 4+1 / q)} d \tau \leq c t^{-1 / q} .
\end{aligned}
$$

Furthermore, (2.10) with $q=2$ shows $\|\nabla u\|_{2} \leq c t^{-1 / 2}$. This implies

$$
\begin{aligned}
\|\nabla u(t)\|_{q} & \leq c t^{-1 / 2}\|u(t / 2)\|_{q}+c \int_{t / 2}^{t}(t-\tau)^{-1 / 2}\|v\|_{2 q /(2-q)}\|\nabla u\|_{2} d \tau \\
& \leq c t^{-1 / 2}+c \int_{t / 2}^{t}(t-\tau)^{-1 / 2} \tau^{-1 / 2-1 / 2} d \tau \leq c t^{-1 / 2} .
\end{aligned}
$$

Combining this with (2.10) gives

$$
\|\nabla u(t)\|_{r} \leq c t^{-1 / 2-(1 / q-1 / r)} \quad(q \leq r \leq 2) .
$$

We further apply (2.10) with $q=2$ to see that if $2<r<\infty$, then

$$
\begin{aligned}
\|\nabla u(t)\|_{r} & \leq c t^{-1 / 2}\|u(t / 2)\|_{r}+c \int_{t / 2}^{t}(t-\tau)^{-1+1 / r}\|v\|_{\infty}\|\nabla u\|_{2} d \tau \\
& \leq c t^{-1 / 2-(1 / q-1 / r)}+c \int_{t / 2}^{t}(t-\tau)^{-1+1 / r} \tau^{-1 / q-1 / 2} d \tau \\
& \leq c t^{-1 / 2-(1 / q-1 / r)} .
\end{aligned}
$$

Choosing $r=4$ and $q=2$ in (2.11), we obtain $\|\nabla u\|_{4} \leq c t^{-3 / 4}$, so that 


$$
\begin{aligned}
\|\nabla u(t)\|_{\infty} & \leq c t^{-1 / 2}\|u(t / 2)\|_{\infty}+c \int_{t / 2}^{t}(t-\tau)^{-3 / 4}\|v\|_{\infty}\|\nabla u\|_{4} d \tau \\
& \leq c t^{-1 / 2-1 / q}+c \int_{t / 2}^{t}(t-\tau)^{-3 / 4} \tau^{-1 / q-3 / 4} d \tau \leq c t^{-1 / 2-1 / q}
\end{aligned}
$$

This proves (i).

Proof of (ii). Let $a \in \boldsymbol{L}_{\sigma}^{2}$ and $(1+|x|) a \in \boldsymbol{L}^{1}\left(\boldsymbol{R}^{2}\right)$. Then (2.5) shows $\|u\|_{2}^{2} \leq c(1+t)^{-2}$. Inserting $s=t / 2$ in (2.9) gives

$$
(t / 2)\|\nabla u(t)\|_{2}^{2} \leq c\|u(t / 2)\|_{2}^{2}\left(1+\|a\|_{2}^{4} \exp \left(c\|a\|_{2}^{4}\right)\right) \leq c(1+t)^{-2} .
$$

Hence,

$$
\|u(t)\|_{\mathrm{BMO}} \leq c\|\nabla u(t)\|_{2} \leq c t^{-3 / 2}
$$

and so

$$
\|u(t)\|_{r} \leq c\|u(t)\|_{\mathrm{BMO}}^{1-2 / r}\|u(t)\|_{2}^{2 / r} \leq c t^{-1 / 2-(1-1 / r)} \quad(2<r<\infty) .
$$

Applying (2.10) with $q=2$, we estimate as

$$
\begin{aligned}
\|u(t)\|_{\infty} & \leq c t^{-1}\|u(t / 2)\|_{1}+c \int_{t / 2}^{t}(t-\tau)^{-3 / 4}\|v\|_{4}\|\nabla u\|_{2} d \tau \\
& \leq c t^{-3 / 2}+c \int_{t / 2}^{t}(t-\tau)^{-3 / 4} \tau^{-5 / 4-1 / 2} d \tau \leq c t^{-3 / 2} .
\end{aligned}
$$

On the other hand, since $\|v\|_{2} \leq\|a\|_{2}$ by (2.3), it follows from (2.12) that

$$
\begin{aligned}
\|\nabla u(t)\|_{1} & \leq c t^{-1 / 2}\|u(t / 2)\|_{1}+c \int_{t / 2}^{t}(t-\tau)^{-1 / 2}\|v\|_{2}\|\nabla u\|_{2} d \tau \\
& \leq c t^{-1}+c \int_{t / 2}^{t}(t-\tau)^{-1 / 2} \tau^{-3 / 2} d \tau \leq c t^{-1} .
\end{aligned}
$$

Combining this with the latter estimate of (2.12), we get

$$
\|\nabla u(t)\|_{r} \leq c t^{-1-(1-1 / r)} \quad(1 \leq r \leq 2) .
$$

When $2<r<\infty$, we again apply $\|\nabla u\|_{2} \leq c t^{-1 / 2}$, to obtain

$$
\begin{aligned}
\|\nabla u(t)\|_{r} & \leq c t^{-1 / 2}\|u(t / 2)\|_{r}+c \int_{t / 2}^{t}(t-\tau)^{-1+1 / r}\|v\|_{\infty}\|\nabla u\|_{2} d \tau \\
& \leq c t^{-1-(1-1 / r)}+c \int_{t / 2}^{t}(t-\tau)^{-1+1 / r} \tau^{-2} d \tau \leq c t^{-1-(1-1 / r)} .
\end{aligned}
$$


Finally, since $a \in \boldsymbol{L}^{1}\left(\boldsymbol{R}^{2}\right)$, we know by (i) that $\|v\|_{\infty} \leq c t^{-1}$ and $\|\nabla u\|_{4} \leq c t^{-5 / 4}$. Hence,

$$
\begin{aligned}
\|\nabla u(t)\|_{\infty} & \leq c t^{-1 / 2}\|u(t / 2)\|_{\infty}+c \int_{t / 2}^{t}(t-\tau)^{-3 / 4}\|v\|_{\infty}\|\nabla u\|_{4} d \tau \\
& \leq c t^{-2}+c \int_{t / 2}^{t}(t-\tau)^{-3 / 4} \tau^{-9 / 4} d \tau \leq c t^{-2} .
\end{aligned}
$$

This proves (ii). The proof of Lemma 2.1 is complete.

We next deduce some weighted $L^{2}$-estimates for approximate solutions. Here we consider approximation scheme (2.1) in the form

$$
\partial_{t} u+v \cdot \nabla u=\Delta u-\nabla p, \quad \nabla \cdot u=0,\left.\quad u\right|_{t=0}=a,
$$

where $u=u^{n}, v=u^{n-1}$, and $p=p^{n}$ is a pressure associated with $u$.

Theorem 2.2. Let $a \in \boldsymbol{L}_{\sigma}^{2},(1+|x|) a \in \boldsymbol{L}^{1}\left(\boldsymbol{R}^{2}\right)$ and $|x|^{\alpha} a \in \boldsymbol{L}^{2}\left(\boldsymbol{R}^{2}\right)$ for some $1<\alpha<2$. Define

$$
g_{\alpha}(t)=(1+t)^{-2 / \alpha}+(1+t)^{-1 / \alpha} t^{-1 / 2} .
$$

Then

$$
\begin{aligned}
& \left\||x|^{\alpha} u^{n}(t)\right\|_{2}^{2}+\int_{0}^{t}\left\||x|^{\alpha} \nabla u^{n}\right\|_{2}^{2} d \tau \\
& \quad \leq c\left(1+\left\||x|^{\alpha} a\right\|_{2}^{2}\right)\left(1+\int_{0}^{\infty} g_{\alpha}(\tau) d \tau\right) \exp \left(c \int_{0}^{\infty} g_{\alpha}(\tau) d \tau\right)
\end{aligned}
$$

for all $t>0$ and $n$, with $c>0$ independent of $n$.

Proof. We multiply $(2.13)$ by $2|x|^{2 \alpha} u$ and integrate by parts, to get

$$
\begin{aligned}
& \frac{d}{d t}\left\||x|^{\alpha} u\right\|_{2}^{2}+2\left\||x|^{\alpha} \nabla u\right\|_{2}^{2} \\
& \quad=\int|u|^{2} \Delta|x|^{2 \alpha} d x+\int|u|^{2}\left(v \cdot \nabla|x|^{2 \alpha}\right) d x+2 \int p u \cdot \nabla|x|^{2 \alpha} d x \\
& \quad \leq c_{\alpha}\left(\int|u|^{2}|x|^{2 \alpha-2} d x+\int|u|^{2}|v||x|^{2 \alpha-1} d x+\int|p||u||x|^{2 \alpha-1} d x\right) \\
& \quad \equiv I_{1}+I_{2}+I_{3} .
\end{aligned}
$$

It is easy to see that 


$$
\begin{aligned}
I_{1} & =c_{\alpha} \int\left(|x|^{\alpha}|u|\right)^{2(\alpha-1) / \alpha}|u|^{2 / \alpha} d x \leq c\left\||x|^{\alpha} u\right\|_{2}^{2(\alpha-1) / \alpha}\|u\|_{2}^{2 / \alpha} \\
& \leq c\left(1+\left\||x|^{\alpha} u\right\|_{2}^{2}\right)\|u\|_{2}^{2 / \alpha} .
\end{aligned}
$$

Consider next

$$
I_{3} \leq c_{\alpha}\left\||x|^{\alpha} u\right\|_{2}\left\||x|^{\alpha-1} p\right\|_{2} .
$$

Since $p=\int\left(\nabla^{2} \mathscr{N}\right)(x-y) \cdot(v \otimes u)(y, t) d y$, with $\mathscr{N}(x)=(2 \pi)^{-1} \log (1 /|x|)$, and since $1<\alpha<2$, the weighted $L^{2}$-estimates for singular integrals ([19]) can be applied to obtain

$$
\begin{aligned}
\left\||x|^{\alpha-1} p\right\|_{2} & \leq c\left\||x|^{\alpha-1}(v \otimes u)\right\|_{2} \\
& \leq c\left(\int\left(|x|^{\alpha}|u|\right)^{2(\alpha-1) / \alpha}|u|^{2 / \alpha}|v|^{2} d x\right)^{1 / 2} \\
& \leq c\left\||x|^{\alpha} u\right\|_{2}^{(\alpha-1) / \alpha}\|u\|_{4}^{1 / \alpha}\|v\|_{4 \alpha} .
\end{aligned}
$$

Therefore,

$$
I_{3} \leq c\left\||x|^{\alpha} u\right\|_{2}^{(2 \alpha-1) / \alpha}\|u\|_{4}^{1 / \alpha}\|v\|_{4 \alpha} \leq c\left(1+\left\||x|^{\alpha} u\right\|_{2}^{2}\right)\|u\|_{4}^{1 / \alpha}\|v\|_{4 \alpha} .
$$

Similarly, we obtain

$$
\begin{aligned}
I_{2} & \leq c_{\alpha} \int|x|^{2 \alpha-1}|u|^{2}|v| d x=c_{\alpha} \int\left(|x|^{\alpha}|u|\right)^{(2 \alpha-1) / \alpha}|u|^{1 / \alpha}|v| d x \\
& \leq c_{\alpha}\left\||x|^{\alpha} u\right\|_{2}^{(2 \alpha-1) / \alpha}\|u\|_{4}^{1 / \alpha}\|v\|_{4 \alpha} \\
& \leq c\left(1+\left\||x|^{\alpha} u\right\|_{2}^{2}\right)\|u\|_{4}^{1 / \alpha}\|v\|_{4 \alpha} .
\end{aligned}
$$

Therefore, (2.14) gives

$$
\frac{d}{d t}\left(1+\left\||x|^{\alpha} u\right\|_{2}^{2}\right)+2\left\||x|^{\alpha} \nabla u\right\|_{2}^{2} \leq c f_{\alpha}(t)\left(1+\left\||x|^{\alpha} u\right\|_{2}^{2}\right)
$$

where

$$
f_{\alpha}(t)=\|u\|_{2}^{2 / \alpha}+\|u\|_{4}^{1 / \alpha}\|v\|_{4 \alpha} .
$$

By Gronwall's lemma, we obtain

$$
1+\left\||x|^{\alpha} u(t)\right\|_{2}^{2} \leq\left(1+\left\||x|^{\alpha} a\right\|_{2}^{2}\right) \exp \left(c \int_{0}^{t} f_{\alpha}(\tau) d \tau\right) .
$$

We estimate $f_{\alpha}$. Since $|x| a \in \boldsymbol{L}^{1}\left(\boldsymbol{R}^{2}\right)$, Lemma 2.1 gives $\|u(t)\|_{2} \leq c(1+t)^{-1}$; so we get 


$$
\|u(t)\|_{2}^{2 / \alpha} \leq c(1+t)^{-2 / \alpha} \quad(1<\alpha<2) .
$$

Secondly, since $\|u\|_{\infty} \leq c t^{-1 / 2}$ and $\|v\|_{\infty} \leq c t^{-1 / 2}$ because $a \in \boldsymbol{L}_{\sigma}^{2}$, we have

$$
\begin{aligned}
& \|u(t)\|_{4}^{1 / \alpha} \leq\|u\|_{2}^{1 / 2 \alpha}\|u\|_{\infty}^{1 / 2 \alpha} \leq c(1+t)^{-1 / 2 \alpha} t^{-1 / 4 \alpha}, \\
& \|v(t)\|_{4 \alpha} \leq\|v\|_{2}^{1 / 2 \alpha}\|v\|_{\infty}^{1-1 / 2 \alpha} \leq c(1+t)^{-1 / 2 \alpha} t^{-1 / 2+1 / 4 \alpha},
\end{aligned}
$$

to obtain

$$
\|u(t)\|_{4}^{1 / \alpha}\|v(t)\|_{4 \alpha} \leq c(1+t)^{-1 / \alpha} t^{-1 / 2} .
$$

The constants $c$ in (2.17) and (2.18) are independent of $n$. Obviously, $f_{\alpha} \leq c g_{\alpha}$; and the assumption $1<\alpha<2$ ensures $\int_{0}^{\infty} g_{\alpha}(\tau) d \tau<\infty$. Thus, from (2.16) we obtain

$$
1+\left\||x|^{\alpha} u(t)\right\|_{2}^{2} \leq\left(1+\left\||x|^{\alpha} a\right\|_{2}^{2}\right) \exp \left(c \int_{0}^{\infty} g_{\alpha}(\tau) d \tau\right) .
$$

Integrating (2.15) now gives

$$
\begin{aligned}
2 \int_{0}^{t}\left\||x|^{\alpha} \nabla u\right\|_{2}^{2} d \tau & \leq 1+\left\||x|^{\alpha} a\right\|_{2}^{2}+c \int_{0}^{t} g_{\alpha}(\tau)\left(1+\left\||x|^{\alpha} u\right\|_{2}^{2}\right) d \tau \\
& \leq 1+\left\||x|^{\alpha} a\right\|_{2}^{2}+c\left(1+\left\||x|^{\alpha} a\right\|_{2}^{2}\right) \exp \left(c \int_{0}^{\infty} g_{\alpha}(\tau) d \tau\right) \int_{0}^{\infty} g_{\alpha}(\tau) d \tau \\
& \leq c\left(1+\left\||x|^{\alpha} a\right\|_{2}^{2}\right)\left(1+\int_{0}^{\infty} g_{\alpha}(\tau) d \tau\right) \exp \left(c \int_{0}^{\infty} g_{\alpha}(\tau) d \tau\right)
\end{aligned}
$$

The proof of Theorem 2.2 is complete.

Now we apply Theorem 2.2 to pointwise estimates for approximate solutions. To this end, we write (2.2) as

$$
u(t)=E_{t} * a-\int_{0}^{t}(\nabla V)(t-\tau) *(v \otimes u)(\tau) d \tau \equiv J_{1}+J_{2} .
$$

Theorem 2.3. Let $a \in \boldsymbol{L}^{\infty}\left(\boldsymbol{R}^{2}\right) \cap \boldsymbol{L}^{1}\left(\boldsymbol{R}^{2}\right), \quad \nabla \cdot a=0, \quad|x|^{\alpha} a \in \boldsymbol{L}^{2}\left(\boldsymbol{R}^{2}\right) \cap$ $\boldsymbol{L}^{2 /(2-\alpha)}\left(\boldsymbol{R}^{2}\right)$ for some $1<\alpha<2$, and $|x| a \in \boldsymbol{L}^{1}\left(\boldsymbol{R}^{2}\right)$. Then

$$
u^{n} \in L^{\infty}\left(\boldsymbol{R}^{2} \times(0, \infty)\right) \quad \text { and } \quad t^{1-\alpha / 2}\left\||x|^{\alpha} u^{n}(t)\right\|_{\infty} \leq c,
$$

with $c>0$ depending only on the norms of $a$ in the spaces as mentioned above.

Proof. Let $2<q \leq \infty$. Since $\|\nabla V(t)\|_{r}=c_{r} t^{-1 / 2-(1-1 / r)}$ for $1 \leq r \leq \infty$, applying Young's inequality for the convolution to (2.2), we see that if $0<$ $t \leq T$, then 


$$
\begin{aligned}
\|u(t)\|_{q} & \leq c_{q}\|a\|_{q}+c_{q} \sup _{[0, T]}\|v\|_{q} \sup _{[0, T]}\|u\|_{q} \int_{0}^{t}(t-\tau)^{-1 / 2-1 / q} d \tau \\
& \leq c_{q}\left(\|a\|_{q}+T^{1 / 2-1 / q} \sup _{[0, T]}\|v\|_{q} \sup _{[0, T]}\|u\|_{q}\right)
\end{aligned}
$$

and so

$$
\sup _{[0, T]}\|u\|_{q} \leq c_{q}\left(\|a\|_{q}+T^{1 / 2-1 / q} \sup _{[0, T]}\|v\|_{q} \sup _{[0, T]}\|u\|_{q}\right) .
$$

Since $1 / 2-1 / q>0$, we can take $T_{q}>0$ and $c_{q}^{\prime}>0$ so that $\|u(t)\|_{q} \leq c_{q}^{\prime}$ for all $t \in\left[0, T_{q}\right]$ uniformly in $n$; see [6] for the details. This, together with Lemma 2.1, gives

$$
\|u(t)\|_{r} \leq c_{r}(1+t)^{-1 / 2-(1-1 / r)} \quad(1 \leq r \leq \infty),
$$

with $c_{r}$ independent of $n$. Now, from $|x|^{\alpha} \leq c_{\alpha}\left(|x-y|^{\alpha}+|y|^{\alpha}\right)$ we have

$$
\left.|| x\right|^{\alpha} J_{1}\left|\leq c \int\right| x-\left.y\right|^{\alpha} E_{t}(x-y)|a(y)| d y+c \int E_{t}(x-y)|y|^{\alpha}|a(y)| d y .
$$

Applying $|x|^{\alpha} E_{t}(x) \leq c t^{-1+\alpha / 2}$, we get

$$
\left.|| x\right|^{\alpha} J_{1}\left|\leq c t^{-1+\alpha / 2}\|a\|_{1}+c\left\|E_{t}\right\|_{2 / \alpha}\left\||x|^{\alpha} a\right\|_{2 /(2-\alpha)} \leq c t^{-1+\alpha / 2} .\right.
$$

In what follows we systematically invoke (2.19). Let $0<\delta<1 / 2$ and decompose $J_{2}$ as

$$
J_{2}=\left(\int_{0}^{t(1-\delta)}+\int_{t(1-\delta)}^{t}\right)(\nabla V)(t-\tau) *(v \otimes u)(\tau) d \tau \equiv J_{21}^{\prime}+J_{22}^{\prime} .
$$

Since $\|u\|_{\infty} \leq c(1+t)^{-3 / 2} \leq c t^{-1+\alpha / 2}$ by $\quad(2.19)$ and since $|x|^{\alpha}|\nabla V(x, t)| \leq$ $c t^{-3 / 2+\alpha / 2}$, we get

$$
\begin{aligned}
\left.|| x\right|^{\alpha} J_{21}^{\prime} \mid \leq & c \int_{0}^{t(1-\delta)} \int|x-y|^{\alpha}|\nabla V(x-y, t-\tau)||u||v| d y d \tau \\
& +\left.c \int_{0}^{t(1-\delta)} \int|\nabla V(x-y, t-\tau)||| y\right|^{\alpha} v|| u \mid d y d \tau \\
\leq & c_{\delta} t^{-1+\alpha / 2} \int_{0}^{t(1-\delta)}(t-\tau)^{-1 / 2}\|u\|_{2}\|v\|_{2} d \tau \\
& +c \int_{0}^{t(1-\delta)}(t-\tau)^{-1}\left\||y|^{\alpha} v\right\|_{2}\|u\|_{\infty} d \tau
\end{aligned}
$$




$$
\begin{aligned}
& \leq c_{\delta} t^{-1+\alpha / 2}\left(1+\left(\sup _{t \geq 0}\left\||x|^{\alpha} v\right\|_{2}\right) \int_{0}^{t(1-\delta)}(t-\tau)^{-\alpha / 2} \tau^{-1+\alpha / 2} d \tau\right) \\
& \leq c_{\delta} t^{-1+\alpha / 2}
\end{aligned}
$$

Here we have used that $\|u\|_{2} \leq c t^{-1 / 2},\|v\|_{2} \leq\|a\|_{2}$ and $\left\||x|^{\alpha} v\right\|_{2} \leq c$. On the other hand, assuming $0 \leq t \leq T$ we have

$$
\begin{aligned}
\left.|| x\right|^{\alpha} J_{22}^{\prime} \mid \leq & c \int_{t(1-\delta)}^{t}\left\||x|^{\alpha} \nabla V(t-\tau)\right\|_{4 / 3}\|u\|_{8}\|v\|_{8} d \tau \\
& +c \int_{t(1-\delta)}^{t}\|\nabla V(t-\tau)\|_{4 / 3}\left\||y|^{\alpha} v\right\|_{\infty}\|u\|_{4} d \tau \\
\leq & c t^{\alpha / 2} \int_{t(1-\delta)}^{t}(t-\tau)^{-3 / 4} \tau^{-5 / 4} d \tau \\
& +c t^{-1+\alpha / 2}\left(\sup _{[0, T]} t^{1-\alpha / 2}\left\||x|^{\alpha} v\right\|_{\infty}\right) \int_{t(1-\delta)}^{t}(t-\tau)^{-3 / 4} \tau^{-1 / 4} d \tau \\
\leq & c_{\delta} t^{-1+\alpha / 2}+c t^{-1+\alpha / 2}\left(\sup _{[0, T]} t^{1-\alpha / 2}\left\||x|^{\alpha} v\right\|_{\infty}\right) \int_{t(1-\delta)}^{t}(t-\tau)^{-3 / 4} \tau^{-1 / 4} d \tau
\end{aligned}
$$

Combining this with (2.20) and (2.21) gives

$$
\begin{gathered}
\sup _{[0, T]} t^{1-\alpha / 2}\left\||x|^{\alpha} u\right\|_{\infty} \leq c_{\delta}+c \chi(\delta) \sup _{[0, T]} t^{1-\alpha / 2}\left\||x|^{\alpha} v\right\|_{\infty} \\
\chi(\delta)=\int_{1-\delta}^{1}(1-\tau)^{-3 / 4} \tau^{-1 / 4} d \tau
\end{gathered}
$$

with $c>0$ independent of $\delta, T$ and $n$. Fixing $\delta>0$ so that $0<c \chi(\delta)<1 / 2$, we deduce

$$
\sup _{[0, T]} t^{1-\alpha / 2}\left\||x|^{\alpha} u^{n}\right\|_{\infty} \leq c^{\prime}+2^{-1} \sup _{[0, T]} t^{1-\alpha / 2}\left\||x|^{\alpha} u^{n-1}\right\|_{\infty}
$$

with $c^{\prime}>0$ independent of $T$ and $n$. Since $J_{1}=u^{0},(2.20)$ and (2.22) together imply

$$
\sup _{t \geq 0} t^{1-\alpha / 2}\left\||x|^{\alpha} u^{n}\right\|_{\infty} \leq c \quad \text { uniformly in } n,
$$

and the proof of Theorem 2.3 is complete.

Proof of Theorem 1.1. Since $\nabla \cdot a=0$ and $a \in \boldsymbol{L}^{1}\left(\boldsymbol{R}^{2}\right)$, we have ([14], [15]) $\int a(y) d y=0 . \quad$ Therefore, 


$$
\begin{aligned}
J_{1}= & \left(\int_{|y| \leq|x| / 2}+\int_{|y| \geq|x| / 2}\right)\left[E_{t}(x-y)-E_{t}(x)\right] a(y) d y \\
= & -\int_{0}^{1} \int_{|y| \leq|x| / 2}\left(y \cdot \nabla E_{t}\right)(x-y \theta) a(y) d y d \theta \\
& +\int_{|y| \geq|x| / 2} E_{t}(x-y) a(y) d y-E_{t}(x) \int_{|y| \geq|x| / 2} a(y) d y \\
\equiv & J_{11}+J_{12}+J_{13} .
\end{aligned}
$$

Since $|x|^{2} E_{t}(x) \leq c,|x|^{3}\left|\nabla E_{t}(x)\right| \leq c$ and $\int E_{t}(x) d x=1$, the assumptions on $a$ give

$$
\begin{aligned}
& \left|J_{11}\right| \leq c \int_{|y| \leq|x| / 2}|x-y \theta|^{-3}|y||a(y)| d y \leq c|x|^{-3}\||x| a\|_{1} \leq c c_{1}|x|^{-3}, \\
& \left|J_{12}\right| \leq c_{0} \int_{|y| \geq|x| / 2} E_{t}(x-y)(1+|y|)^{-3} d y \leq c c_{0}(1+|x|)^{-3} \\
& \left|J_{13}\right| \leq c c_{0}|x|^{-2} \int_{|y| \geq|x| / 2}(1+|y|)^{-3} d y \leq c c_{0}|x|^{-3} .
\end{aligned}
$$

We thus obtain

$$
\left\||x|^{3} J_{1}\right\|_{\infty} \leq c\left(c_{0}+c_{1}\right)
$$

On the other hand,

$$
\begin{aligned}
\left.|| x\right|^{3} J_{2} \mid \leq & c \int_{0}^{t} \int|x-y|^{3}|\nabla V(x-y, t-\tau)||(v \otimes u)(y, \tau)| d y d \tau \\
& +c \int_{0}^{t} \int|\nabla V(x-y, t-\tau)||y|^{3}(v \otimes u)(y, \tau) \mid d y d \tau \\
\equiv & J_{21}^{\prime \prime}+J_{22}^{\prime \prime} .
\end{aligned}
$$

Applying $|x|^{3}|\nabla V(x, t)| \leq c,\|u(t)\|_{2} \leq c(1+t)^{-1}$ and $\|v(t)\|_{2} \leq c(1+t)^{-1}$, we get

$$
J_{21}^{\prime \prime} \leq c \int_{0}^{t}\|u\|_{2}\|v\|_{2} d \tau \leq c\left(\int_{0}^{\infty}\|u\|_{2}^{2} d \tau\right)^{1 / 2}\left(\int_{0}^{\infty}\|v\|_{2}^{2} d \tau\right)^{1 / 2}=c .
$$

Furthermore, we write

$$
J_{22}^{\prime \prime}=\left(\int_{0}^{t(1-\delta)}+\int_{t(1-\delta)}^{t}\right) \int|\nabla V(x-y, t-\tau)||y|^{3}|(v \otimes u)(y, \tau)| d y d \tau \equiv J_{221}^{\prime \prime}+J_{222}^{\prime \prime}
$$


Theorem 2.3 ensures $\left\||y|^{3 / 2} u\right\|_{\infty} \leq c \tau^{-1 / 4}$ and $\left\||y|^{3 / 2} v\right\|_{\infty} \leq c \tau^{-1 / 4}$ uniformly in n. Therefore,

$$
J_{221}^{\prime \prime} \leq c \int_{0}^{t(1-\delta)}(t-\tau)^{-1 / 2}\left\||y|^{3 / 2} u\right\|_{\infty}\left\||y|^{3 / 2} v\right\|_{\infty} d \tau \leq c \int_{0}^{t}(t-\tau)^{-1 / 2} \tau^{-1 / 2} d \tau=c .
$$

Moreover, assuming $0 \leq t \leq T$ we get

$$
J_{222}^{\prime \prime} \leq c \sup _{[0, T]}\left\||y|^{3} v(t)\right\|_{\infty} \int_{t(1-\delta)}^{t}(t-\tau)^{-1 / 2}\|u(\tau)\|_{\infty} d \tau .
$$

Since $a \in \boldsymbol{L}_{\sigma}^{2}$, Lemma 2.1 shows $\|u(t)\|_{\infty} \leq c t^{-1 / 2}$ uniformly in $n$; and so

$$
J_{222}^{\prime \prime} \leq c \psi(\delta) \sup _{[0, T]}\left\||y|^{3} v(t)\right\|_{\infty}, \quad \psi(\delta)=\int_{1-\delta}^{1}(1-\tau)^{-1 / 2} \tau^{-1 / 2} d \tau .
$$

Collecting terms gives

$$
\sup _{[0, T]}\left\||x|^{3} u(t)\right\|_{\infty} \leq c_{1}+c_{2} \psi(\delta) \sup _{[0, T]}\left\||y|^{3} v(t)\right\|_{\infty},
$$

with $c_{1}$ and $c_{2}$ independent of $T, n$ and $\delta$. Fixing $\delta$ so that $0<c_{2} \psi(\delta)<1 / 2$, we deduce

$$
\sup _{[0, T]}\left\||x|^{3} u^{n}(t)\right\|_{\infty} \leq c_{3}+2^{-1} \sup _{[0, T]}\left\||x|^{3} u^{n-1}(t)\right\|_{\infty},
$$

with $c_{3}>0$ independent of $n$ and $T$. Since $J_{1}=u^{0},(2.23)$ and (2.24) together yield

$$
\sup _{t \geq 0}\left\||x|^{3} u^{n}(t)\right\|_{\infty} \leq c_{4} \quad \text { uniformly in } n .
$$

Combining this with (2.19) yields

$$
\left|u^{n}(x, t)\right| \leq\left\{\begin{array}{l}
c(1+|x|)^{-3}, \\
c(1+t)^{-3 / 2},
\end{array} \quad\left\|u^{n}(t)\right\|_{r} \leq c(1+t)^{-1 / 2-(1-1 / r)} \quad(1 \leq r \leq \infty),\right.
$$

uniformly in $n$. Theorem 1.1 is now deduced by passing to the limit as $n \rightarrow \infty$, as shown in the next lemma. This completes the proof.

Lemma 2.4. Let $a \in \boldsymbol{L}_{\sigma}^{2}$ and let $u$ be the solution corresponding to the initial velocity $a$. Then the approximate solutions defined by (2.1) or (2.2) converge to $u$ in $B C\left([0, \infty): \boldsymbol{L}_{\sigma}^{2}\right)$.

Remark. In the theory of Navier-Stokes equations, we can in general only show that approximate solutions contain a subsequence converging to the desired solution. However, contrary to the other works, our approximate solutions are defined by (2.1) or (2.2); namely, the convection term $u \cdot \nabla u$ is 
replaced not with $u^{n} \cdot \nabla u^{n}$, but with $u^{n-1} \cdot \nabla u^{n}$. In this case, we cannot expect the existence of a convergent subsequence. Therefore, in order to complete our argument in this section, we have to show that the sequence $\left\{u^{n}\right\}$ itself converges to the desired solution. Lemma 2.4 above gives an affirmative answer to this question in the case of two space dimensions.

Proof of Lemma 2.4. Consider (2.1):

$$
\partial_{t} u^{n}+A u^{n}=-P\left(u^{n-1} \cdot \nabla u^{n}\right), \quad u^{n}(0)=a \quad\left(u^{-1}=0\right) .
$$

Let $u$ be the (unique) solution with $u(0)=a$, and define $w^{n}=u^{n}-u$ for $n=$ $0,1,2, \ldots$ The energy conservation (2.3) for $u$ and $u^{n}$ implies

$$
\|u(t)\|_{2} \leq\|a\|_{2} ; \quad \text { and } \quad\left\|u^{n}(t)\right\|_{2} \leq\|a\|_{2} \quad \text { for all } t>0 \text { and } n \text {. }
$$

Direct calculation gives

$$
\partial_{t} w^{n}+A w^{n}=-P\left(u^{n-1} \cdot \nabla w^{n}\right)-P\left(w^{n-1} \cdot \nabla u\right) \quad \text { and } \quad w^{n}(0)=0 .
$$

Since $\left(u^{n-1} \cdot \nabla w^{n}, w^{n}\right)=0$, with $(\cdot, \cdot)$ the $L^{2}$-inner product, we have

$$
\frac{d}{d t}\left\|w^{n}\right\|_{2}^{2}+2\left\|\nabla w^{n}\right\|_{2}^{2}=-2\left(w^{n-1} \cdot \nabla u, w^{n}\right)=2\left(w^{n-1} \cdot \nabla w^{n}, u\right) .
$$

By duality between the Hardy space $\mathscr{H}^{1}$ and BMO ([19]) and the estimate $\|u\|_{\text {BMO }} \leq c\|\nabla u\|_{2}$, we obtain

$$
\begin{aligned}
2\left|\left(w^{n-1} \cdot \nabla w^{n}, u\right)\right| & \leq 2\left\|w^{n-1} \cdot \nabla w^{n}\right\|_{\mathscr{H}^{1}}\|u\|_{\text {BMO }} \leq c\left\|w^{n-1}\right\|_{2}\left\|\nabla w^{n}\right\|_{2}\|\nabla u\|_{2} \\
& \leq\left\|\nabla w^{n}\right\|_{2}^{2}+c_{0}\|\nabla u\|_{2}^{2}\left\|w^{n-1}\right\|_{2}^{2}
\end{aligned}
$$

with $c_{0}>0$ independent of $n$ and $u$. Here we have applied ([4], [15])

$$
\left\|w^{n-1} \cdot \nabla w^{n}\right\|_{\mathscr{H}^{1}} \leq c\left\|w^{n-1}\right\|_{2}\left\|\nabla w^{n}\right\|_{2} .
$$

Therefore, (2.26) gives

$$
\frac{d}{d t}\left\|w^{n}\right\|_{2}^{2}+\left\|\nabla w^{n}\right\|_{2}^{2} \leq c_{0}\|\nabla u\|_{2}^{2}\left\|w^{n-1}\right\|_{2}^{2} .
$$

Integrating (2.27) over $[0, t] \subset[0, T]$ and noting that $w^{n}(0)=0$, we obtain

$$
\left\|w^{n}(t)\right\|_{2}^{2}+\int_{0}^{t}\left\|\nabla w^{n}\right\|_{2}^{2} d \tau \leq c_{0} \sup _{[0, T]}\left\|w^{n-1}\right\|_{2}^{2} \int_{0}^{T}\|\nabla u\|_{2}^{2} d \tau .
$$

Hence,

$$
\sup _{[0, T]}\left\|w^{n}\right\|_{2}^{2} \leq c_{0} \sup _{[0, T]}\left\|w^{n-1}\right\|_{2}^{2} \int_{0}^{T}\|\nabla u\|_{2}^{2} d \tau
$$


Here we fix $T>0$ so that

$$
c_{0} \int_{s}^{s+T}\|\nabla u\|_{2}^{2} d \tau<1 / 4 \quad \text { for all } s \geq 0
$$

which is possible since $\|\nabla u\|_{2} \in L^{2}(0, \infty)$ and $E \mapsto \int_{E}\|\nabla u\|_{2}^{2} d \tau$ is absolutely continuous with respect to Lebesgue measure. We thus obtain, by (2.25),

$$
\sup _{[0, T]}\left\|w^{n}\right\|_{2} \leq 2^{-1} \sup _{[0, T]}\left\|w^{n-1}\right\|_{2} \leq 2^{-n} \sup _{[0, T]}\left\|u^{0}-u\right\|_{2} \leq 2^{1-n}\|a\|_{2}
$$

and so,

$$
\lim _{n \rightarrow \infty} \sup _{[0, T]}\left\|u^{n}-u\right\|_{2}=0 .
$$

We next integrate (2.27) over $[T / 2, t] \subset[T / 2,3 T / 2]$ to get

$$
\left\|w^{n}(t)\right\|_{2}^{2}+\int_{T / 2}^{t}\left\|\nabla w^{n}\right\|_{2}^{2} d \tau \leq\left\|w^{n}(T / 2)\right\|_{2}^{2}+c_{0} \sup _{[T / 2,3 T / 2]}\left\|w^{n-1}\right\|_{2}^{2} \int_{T / 2}^{T}\|\nabla u\|_{2}^{2} d \tau,
$$

so that, by (2.28),

$$
\sup _{[T / 2,3 T / 2]}\left\|w^{n}\right\|_{2} \leq\left\|w^{n}(T / 2)\right\|_{2}+2^{-1} \sup _{[T / 2,3 T / 2]}\left\|w^{n-1}\right\|_{2} .
$$

Given $\varepsilon>0$, choose $N$ such that

$$
\left\|w^{n}(T / 2)\right\|_{2}<\varepsilon \quad \text { whenever } n \geq N .
$$

This is possible by (2.29). It follows from (2.25) that

$$
\begin{aligned}
\sup _{[T / 2,3 T / 2]}\left\|w^{n}\right\|_{2} & <\varepsilon+2^{-1} \sup _{[T / 2,3 T / 2]}\left\|w^{n-1}\right\|_{2} \\
& <\left(1+2^{-1}\right) \varepsilon+2^{-2} \sup _{[T / 2,3 T / 2]}\left\|w^{n-2}\right\|_{2} \\
& <2 \varepsilon+2^{N-n} \sup _{[T / 2,3 T / 2]}\left\|w^{N}\right\|_{2} \\
& \leq 2 \varepsilon+2^{N-n+1}\|a\|_{2}
\end{aligned}
$$

whenever $n>N$. Hence,

$$
\limsup _{n \rightarrow \infty}\left(\sup _{[T / 2,3 T / 2]}\left\|u^{n}-u\right\|_{2}\right) \leq 2 \varepsilon .
$$

Since $\varepsilon>0$ was arbitrary, we conclude that

$$
\lim _{n \rightarrow \infty} \sup _{[T / 2,3 T / 2]}\left\|u^{n}-u\right\|_{2}=0 .
$$


Repeating these processes eventually shows that

$$
\lim _{n \rightarrow \infty} \sup _{[j T / 2,(j+2) T / 2]}\left\|u^{n}-u\right\|_{2}=0 \quad \text { for every } j=1,2, \ldots
$$

So the sequence $\left\{u^{n}\right\}$ itself converges to the solution $u$ in $C\left([0, T]: \boldsymbol{L}_{\sigma}^{2}\right)$ for any fixed finite interval $[0, T]$.

On the other hand, the energy conservation for $u$ implies $\int_{0}^{\infty}\|\nabla u\|_{2}^{2} d \tau<\infty$, and so for each $\varepsilon>0$ there is a number $M>0$ so that

$$
2 c_{0}\|a\|_{2}^{2} \int_{M}^{\infty}\|\nabla u\|_{2}^{2} d \tau<\varepsilon \quad \text { whenever } t>M
$$

Due to $(2.25)$, we have

$$
\begin{aligned}
\left\|w^{n}(t)\right\|_{2}^{2} & \leq \sup _{[0, M]}\left\|w^{n}\right\|_{2}^{2}+c_{0} \sup _{[M, t]}\left\|w^{n}\right\|_{2}^{2} \int_{M}^{\infty}\|\nabla u\|_{2}^{2} d \tau \\
& \leq \sup _{[0, M]}\left\|w^{n}\right\|_{2}^{2}+2 c_{0}\|a\|_{2}^{2} \int_{M}^{\infty}\|\nabla u\|_{2}^{2} d \tau \\
& \leq \sup _{[0, M]}\left\|w^{n}\right\|_{2}^{2}+\varepsilon
\end{aligned}
$$

for $t>M$. This implies $\lim \sup _{n \rightarrow \infty}\left(\sup _{t \geq 0}\left\|u^{n}(t)-u(t)\right\|_{2}^{2}\right) \leq \varepsilon$; and so

$$
\lim _{n \rightarrow \infty} \sup _{t \geq 0}\left\|u^{n}(t)-u(t)\right\|_{2}=0
$$

since $\varepsilon>0$ was arbitrary. The proof of Lemma 2.4 is complete.

\section{Proof of Theorem 1.3}

In this section $u$ denotes the solution given in Theorem 1.1. We shall prove (1.12) and (1.13), assuming further that $a$ is $\mathscr{D}_{m+2}$-covariant (see (1.9) for the definition) and

$$
(1+|y|)^{m+3} a \in \boldsymbol{L}^{\infty}\left(\boldsymbol{R}^{2}\right), \quad(1+|y|)^{m+1} a \in \boldsymbol{L}^{1}\left(\boldsymbol{R}^{2}\right) .
$$

The basic idea is to apply Taylor's formula to $y \mapsto E_{t}(x-y)$ and $y \mapsto$ $V(x-y, t-\tau)$, and then to examine the cancellation properties of the resulting Taylor polynomials, making use of the $\mathscr{D}_{m+2}$-covariance of functions $a$ and $u$. To this end, we need a few preliminaries.

Proposition 3.1. Let $s \in N \cup\{0\}$ and $A=\left(A_{j k}\right) \in O(2)$.

(i) If $a$ is covariant with the action of $A$, so is the vector field 


$$
\left(\sum_{|\alpha|=s} \frac{1}{\alpha !}\left(\partial_{x}^{\alpha} V_{j k}\right)(x, t) \int y^{\alpha} a_{k}(y) d y\right)_{j=1}^{2}
$$

for any fixed $t>0$.

(ii) If $u(\cdot, t)$ and $v(\cdot, t)$ are covariant with the action of $A$, so is the vector field

$$
\left(\sum_{|\beta|=s-1} \frac{1}{\beta !}\left(\partial_{\ell} \partial_{x}^{\beta} V_{j k}\right)(x, t) \int y^{\beta}\left(v_{\ell} u_{k}\right)(y, \tau) d y\right)_{j=1}^{2}
$$

for any fixed $t>0$ and $\tau>0$. (The assertion is vacuous when $s=0$.)

Proof. Observe that (see (1.4))

$$
V_{j k}(x, t)=\left(\delta_{j k}+R_{j} R_{k}\right) E_{t}(x),
$$

where $R_{j}$ is the $j$-th Riesz transform ([19]), i.e., the convolution operator with kernel

$$
R_{j}(x)=c \text { p.v. } x_{j} /|x|^{3}, \quad j=1,2 .
$$

Since $E_{t}(A x)=E_{t}(x)$, we have $\left(R_{j} E_{t}\right)(A x)=A_{j k}\left(R_{k} E_{t}\right)(x)$, which implies

$$
V_{j k}(A x, t)=A_{j \ell} A_{k s} V_{\ell s}(x, t), \quad j, k=1,2 .
$$

Now we prove (i). By the chain rule and the assumption that $A \in O(2)$, we get

$$
\left(\partial_{x}^{\alpha} V_{j k}\right)(A x, t)=\left[(A \nabla)^{\alpha} A_{j \ell} A_{k s} V_{\ell s}\right](x, t), \quad \text { where }(A \nabla)^{\alpha}=\prod_{j}\left(A_{j k} \partial_{k}\right)^{\alpha_{j}} .
$$

Using $d A z=|\operatorname{det} A| d z=d z$, we obtain

$$
\begin{aligned}
\sum_{|\alpha|=s} \frac{1}{\alpha !}\left(\partial_{x}^{\alpha} V_{j k}\right)(A x, t) \int y^{\alpha} a_{k}(y) d y & =\sum_{|\alpha|=s} \frac{1}{\alpha !}\left[(A \nabla)^{\alpha} A_{j \ell} A_{k s} V_{\ell s}\right](x, t) \int(A z)^{\alpha} a_{k}(A z) d z \\
& =\sum_{|\alpha|=s} \frac{1}{\alpha !}\left[(A \nabla)^{\alpha} A_{j \ell} A_{k s} V_{\ell s}\right](x, t) \int(A z)^{\alpha} A_{k h} a_{h}(z) d z \\
& =\sum_{|\alpha|=s} \frac{1}{\alpha !}\left[(A \nabla)^{\alpha} A_{j \ell} V_{\ell h}\right](x, t) \int(A z)^{\alpha} a_{h}(z) d z \\
& =\int \sum_{|\alpha|=s} \frac{1}{\alpha !}(A z)^{\alpha}\left(A \nabla_{x}\right)^{\alpha} A_{j \ell} V_{\ell h}(x, t) a_{h}(z) d z .
\end{aligned}
$$

On the other hand, since $A \in O(2)$, the Binomial Theorem gives 


$$
\sum_{|\alpha|=s} \frac{1}{\alpha !}(A z)^{\alpha}\left(A \nabla_{x}\right)^{\alpha}=\frac{1}{s !}\left(A z \cdot A \nabla_{x}\right)^{s}=\frac{1}{s !}\left(z \cdot \nabla_{x}\right)^{s}=\sum_{|\alpha|=s} \frac{1}{\alpha !} z^{\alpha} \partial_{x}^{\alpha} .
$$

It follows that

$$
\int \sum_{|\alpha|=s} \frac{1}{\alpha !}(A z)^{\alpha}\left(A \nabla_{x}\right)^{\alpha} A_{j \ell} V_{\ell h}(x, t) a_{h}(z) d z=A_{j \ell} \sum_{|\alpha|=s} \frac{1}{\alpha !}\left(\partial_{x}^{\alpha} V_{\ell k}\right)(x, t) \int z^{\alpha} a_{k}(z) d z .
$$

This proves (i). (ii) is proved similarly. The proof of Proposition 3.1 is complete.

Proposition 3.2. Let $m \in N$ and suppose given $a, u(\cdot, t)$ and $v(\cdot, t)$, which are $\mathscr{D}_{n}$-covariant for some $n \geq m+2$, satisfying $(1+|x|)^{m} a \in \boldsymbol{L}^{1}\left(\boldsymbol{R}^{2}\right)$ and $(1+|x|)^{(m-1) / 2} u \in \boldsymbol{L}^{2}\left(\boldsymbol{R}^{2}\right)$. Then, for any fixed $t>0, \tau>0$ and $s \in\{0, \ldots, m\}$, we have

$$
\begin{gathered}
\left(\sum_{|\alpha|=s} \frac{1}{\alpha !}\left(\partial_{x}^{\alpha} V_{j k}\right)(x, t) \int y^{\alpha} a_{k}(y) d y\right)_{j=1}^{2}=(0,0), \\
\left(\sum_{|\beta|=s-1} \frac{1}{\beta !}\left(\partial_{x}^{\beta} \partial_{\ell} V_{j k}\right)(x, t) \int y^{\beta}\left(v_{\ell} u_{k}\right)(y, \tau) d y\right)_{j=1}^{2}=(0,0) .
\end{gathered}
$$

Proof. The idea below is due to [3]. Since $d A_{n} y=\left|\operatorname{det} A_{n}\right| d y=d y$, the $\mathscr{D}_{n}$-covariance of the vector field $a$ gives

$$
\int a(y) d y=\int a\left(A_{n} y\right) d y=\int A_{n} a(y) d y=A_{n} \int a(y) d y .
$$

This shows that $\int a(y) d y$ is an eigenvector of the rotation $A_{n}$ in (1.8) corresponding to eigenvalue 1 . But, since $A_{n}$ has not eigenvalue 1 , this integral vanishes. Hence (3.1) holds for $s=0$; and (3.2) is vacuous when $s=0$. Thus, we may assume $n \geq 3$ and $s \geq 1$.

First we prove (3.1). The Fourier transform of the left-hand side equals

$$
i^{s} e^{-t|\xi|^{2}}\left(\delta_{j k}-\xi_{j} \xi_{k} /|\xi|^{2}\right) \sum_{|\alpha|=s} \frac{\xi^{\alpha}}{\alpha !} \int y^{\alpha} a_{k}(y) d y, \quad j=1,2 .
$$

The Fourier transform, divided by $i^{s+1} e^{-t|\xi|^{2}}$, of the associated vorticity is

$$
\sum_{|\alpha|=S} \frac{\xi^{\alpha}}{\alpha !} \int y^{\alpha}(\xi \times a(y)) d y \quad\left(\xi \times a=\xi_{1} a_{2}-\xi_{2} a_{1}\right) .
$$

This is a homogeneous polynomial of $\xi$ of degree $\leq s+1$ which is $\mathscr{D}_{n}$-covariant in the sense of $(1.10)$. (The $\mathscr{D}_{n}$-covariance of a vorticity in the Fourier image 
is of the same form as (1.10).) We restrict (3.3) to the unit circle $\{\xi:|\xi|=1\}$ and write it in terms of the polar coordinates $\xi_{1}=\cos \theta, \xi_{2}=\sin \theta$, to get a trigonometric polynomial of degree $\leq s+1$ :

$$
a_{0}+\sum_{j=1}^{s+1}\left(a_{j} \cos j \theta+b_{j} \sin j \theta\right) .
$$

The $\mathscr{D}_{n}$-covariance (in the sense of $(1.10)$ ) shows that (3.4) is $2 \pi / n$-periodic, and this implies

$$
\left(\begin{array}{l}
a_{j} \\
b_{j}
\end{array}\right)=\left(\begin{array}{cc}
\cos (2 j \pi / n) & \sin (2 j \pi / n) \\
-\sin (2 j \pi / n) & \cos (2 j \pi / n)
\end{array}\right)\left(\begin{array}{c}
a_{j} \\
b_{j}
\end{array}\right) \quad(j=1, \ldots, s+1) .
$$

Since $1 \leq j \leq s+1 \leq m+1<m+2 \leq n$, the matrices on the right-hand side above have not eigenvalue 1 , and so $a_{j}=b_{j}=0$ for all $j \geq 1$. Therefore, (3.4) must be constant $a_{0}$. Since $a_{0}$ should be covariant (in the sense of (1.10)) with the reflection $J$ given in (1.8), it follows that $a_{0}=0$. Thus, (3.3) vanishes identically. This implies that the left-hand side of (3.1) is harmonic on $\boldsymbol{R}^{2}$, bounded and decays to 0 as $|x| \rightarrow \infty$. Hence we obtain (3.1). Assertion (3.2) is proved in the same way: The Fourier transform, divided by $i^{s+1} e^{-t|\xi|^{2}}$, of the associated vorticity is

$$
\sum_{|\beta|=s-1} \frac{\xi^{\beta}}{\beta !} \int y^{\beta}(\xi \cdot v)(y, \tau)(\xi \times u)(y, \tau) d y,
$$

which is a homogeneous $\mathscr{D}_{n}$-covariant polynomial of degree $\leq s+1$. The remaining part of the proof is completely the same as in the foregoing case; so the proof of Proposition 3.2 is complete.

Corollary 3.3. For $a=\left(a_{j}\right)_{j=1}^{2}$ given in Proposition 3.2, suppose further that $\nabla \cdot a=0$ and $|x|^{m+1} a \in \boldsymbol{L}^{1}\left(\boldsymbol{R}^{2}\right)$. Then

$$
\left(\sum_{|\alpha|=s} \frac{\partial_{x}^{\alpha} E_{t}(x)}{\alpha !} \int y^{\alpha} a_{j}(y) d y\right)_{j=1}^{2}=(0,0) \quad \text { for } s=0, \ldots, m .
$$

Proof. We know ([14], [15]) that $\nabla \cdot a=0$ implies $\int a(y) d y=0$. Hence, Corollary 3.3 is obvious for $s=0$. To treat the case $s \geq 1$, we write (see (1.4))

$$
V_{j k}(x, t)=E_{t}(x) \delta_{j k}+W_{j k}(x, t), \quad W_{j k}(x, t)=\int_{0}^{\infty} \partial_{j} \partial_{k} E_{t+\tau}(x) d \tau,
$$

and find that, since $\nabla \cdot a=0$,

$$
\int W_{j k}(x-y, t) a_{k}(y) d y=0, \quad j=1,2 .
$$


To prove Corollary 3.3 for $s \geq 1$, it suffices (in view of Proposition 3.2) to show that

$$
\left(\sum_{|\alpha|=s} \frac{\partial_{x}^{\alpha} W_{j k}(x, t)}{\alpha !} \int y^{\alpha} a_{k}(y) d y\right)_{j=1}^{2}=(0,0) \quad \text { for } s=1, \ldots, m .
$$

Since $\int a(y) d y=0$, applying Taylor's formula to (3.5) yields

$$
0=\sum_{1 \leq|\alpha| \leq m} \frac{\partial_{x}^{\alpha} W_{j k}(x, t)}{\alpha !} \int(-y)^{\alpha} a_{k}(y) d y+\int W_{j k}^{(m+1)}(x, y, t) a_{k}(y) d y,
$$

where

$$
W_{j k}^{(m+1)}(x, y, t)=(m+1) \int_{0}^{1}(1-\theta)^{m} \sum_{|\beta|=m+1} \frac{(-y)^{\beta}}{\beta !}\left(\partial_{x}^{\beta} W_{j k}\right)(x-y \theta, t) d \theta .
$$

(Note that the last integral in (3.7) makes sense because $|x|^{m+1} a \in \boldsymbol{L}^{1}\left(\boldsymbol{R}^{2}\right)$ by assumption.) Since $|\alpha| \geq 1$, we have

$$
\partial_{x}^{\alpha} W_{j k}(x, t)=t^{-1-|\alpha| / 2} K_{j k, \alpha}(x / \sqrt{t}), \quad \text { with } K_{j k, \alpha} \in L^{1}\left(\boldsymbol{R}^{2}\right) \cap L^{\infty}\left(\boldsymbol{R}^{2}\right) .
$$

(Note that $K_{j k, \alpha} \notin L^{1}\left(\boldsymbol{R}^{2}\right)$ when $\alpha=0$.) We write (3.7) as

$$
\begin{aligned}
& -\sum_{|\alpha|=1} \frac{\partial_{x}^{\alpha} W_{j k}(x, t)}{\alpha !} \int(-y)^{\alpha} a_{k}(y) d y \\
& \quad=\sum_{2 \leq|\alpha| \leq m} \frac{\partial_{x}^{\alpha} W_{j k}(x, t)}{\alpha !} \int(-y)^{\alpha} a_{k}(y) d y+\int W_{j k}^{(m+1)}(x, y, t) a_{k}(y) d y,
\end{aligned}
$$

and take the $L^{1}$-norm of both sides of (3.9). Due to (3.8), the $L^{1}$-norm of the left-hand side is equal to $c_{1} t^{-1 / 2}$ with a constant $c_{1} \geq 0$; so we get $0 \leq$ $c_{1} t^{-1 / 2} \leq c_{1}^{\prime}\left(t^{-1}+\cdots+t^{-(m+1) / 2}\right)$ for all $t>0$. Multiplying both sides by $t^{1 / 2}$ and passing to the limit as $t \rightarrow \infty$, we deduce $c_{1}=0$. Hence (3.6) holds for $s=1$. This also implies

$$
\begin{aligned}
& -\sum_{|\alpha|=2} \frac{\partial_{x}^{\alpha} W_{j k}(x, t)}{\alpha !} \int(-y)^{\alpha} a_{k}(y) d y \\
& \quad=\sum_{3 \leq|\alpha| \leq m} \frac{\partial_{x}^{\alpha} W_{j k}(x, t)}{\alpha !} \int(-y)^{\alpha} a_{k}(y) d y+\int W_{j k}^{(m+1)}(x, y, t) a_{k}(y) d y .
\end{aligned}
$$

In view of (3.8), we obtain $0 \leq c_{2} t^{-1} \leq c_{2}^{\prime}\left(t^{-3 / 2}+\cdots+t^{-(m+1) / 2}\right)$ for all $t>0$, and so $c_{2}=0$. This proves (3.6) for $s=2$. The result now follows by induction. 
Lemma 3.4. If a is $\mathscr{D}_{m}$-covariant for some $m \geq 3$, so is the solution $u(\cdot, t)$ for all $t>0$.

Proof. Recall that the solution $u$ is obtained via approximate solutions $\left\{u^{n}\right\}$ defined by

$$
\begin{gathered}
u^{n}(t)=u^{0}(t)-\int_{0}^{t}(\nabla V)(t-\tau) *\left(u^{n-1} \otimes u^{n}\right)(\tau) d \tau \quad(n \geq 1), \\
u^{0}(t)=E_{t} * a=V(t) * a .
\end{gathered}
$$

We can inductively verify that when $a$ is $\mathscr{D}_{m}$-covariant, the approximate solutions belong to $L^{2}$ Sobolev spaces of $\mathscr{D}_{m}$-covariant vector fields. Indeed, If $a$ and $u^{n-1}$ are $\mathscr{D}_{m}$-covariant, the right-hand side of the first equation above defines an affine operator of $u^{n}$ which leaves invariant the $L^{2}$ Sobolev spaces of $\mathscr{D}_{m}$-covariant fields. This fact is easily verified with the aid of the Fourier transform, and so the details are omitted here.

Proof of Theorem 1.3. We prove (1.12) and (1.13) by induction on $m$. Theorem 1.1 implies

$$
\begin{gathered}
\int|u(y, \tau)|^{2} d y \leq c(1+\tau)^{-2} \\
\int(1+|y|)^{3}|u(y, \tau)|^{2} d y \leq c \int|u(y, \tau)| d y \leq c(1+\tau)^{-1 / 2} .
\end{gathered}
$$

By Hölder's inequality we get

$$
\int(1+|y|)|u(y, \tau)|^{2} d y \leq\left(\int(1+|y|)^{3}|u|^{2} d y\right)^{1 / 3}\left(\int|u|^{2} d y\right)^{2 / 3} \leq c(1+\tau)^{-3 / 2}
$$

and so

$$
\int_{0}^{\infty} \int(1+|y|)|u(y, \tau)|^{2} d y d \tau<\infty
$$

Now, let $m=1$, i.e., let the solution $u$ be $\mathscr{D}_{3}$-covariant and suppose that

$$
(1+|y|)^{4} a \in \boldsymbol{L}^{\infty}\left(\boldsymbol{R}^{2}\right), \quad(1+|y|)^{2} a \in \boldsymbol{L}^{1}\left(\boldsymbol{R}^{2}\right) .
$$

Proposition 3.2 and Corollary 3.3 together imply

$$
\begin{aligned}
& \left(\sum_{|\alpha|=s} \frac{\partial_{x}^{\alpha} E_{t}(x)}{\alpha !} \int y^{\alpha} a_{j}(y) d y\right)_{j=1}^{2}=(0,0), \quad s=0,1, \\
& \left(\partial_{\ell} V_{j k}(x, t) \int\left(u_{\ell} u_{k}\right)(y, \tau) d y\right)_{j=1}^{2}=(0,0) .
\end{aligned}
$$


By (3.10), (3.11) and the assumption that $(1+|y|)^{2} a \in \boldsymbol{L}^{1}\left(\boldsymbol{R}^{2}\right)$, we have

$$
\begin{aligned}
u(x, t)= & \int E_{t}^{(2)}(x, y) a(y) d y-\int_{0}^{t / 2} \int \nabla V^{(1)}(x, y, t-\tau)(u \otimes u)(y, \tau) d y d \tau \\
& -\int_{t / 2}^{t} \nabla V(t-\tau) *(u \otimes u)(\tau) d \tau \equiv I+J_{1}+J_{2} \quad(j=1,2),
\end{aligned}
$$

where

$$
\begin{aligned}
& E_{t}^{(s)}(x, y)=s \int_{0}^{1}(1-\theta)^{s-1} \sum_{|\alpha|=s} \frac{(-y)^{\alpha}}{\alpha !}\left(\partial_{x}^{\alpha} E_{t}\right)(x-y \theta) d \theta, \\
& V^{(s)}(x, y, t)=s \int_{0}^{1}(1-\theta)^{s-1} \sum_{|\beta|=s} \frac{(-y)^{\beta}}{\beta !}\left(\partial_{x}^{\beta} V\right)(x-y \theta, t) d \theta .
\end{aligned}
$$

We first prove

$$
\|u(t)\|_{1} \leq c(1+t)^{-1} .
$$

Indeed, $\left\|\partial_{x}^{2} E_{t}\right\|_{1}=c t^{-1}$ implies $\|I\|_{1} \leq c(1+t)^{-1}$. Moreover, by (3.10) and the fact that $\left\|\partial_{x}^{\alpha} V(\cdot, t)\right\|_{1}=c_{\alpha} t^{-|\alpha| / 2}$ for $|\alpha| \geq 1$, we find

$\left\|J_{1}\right\|_{1} \leq c \int_{0}^{t / 2}(t-\tau)^{-1}\left\|(1+|y|)^{1 / 2} u\right\|_{2}^{2} d \tau \leq c t^{-1} \int_{0}^{\infty} \int(1+|y|)|u(y, \tau)|^{2} d y d \tau=c t^{-1}$.

Since $\|u(\tau)\|_{2}^{2} \leq c(1+\tau)^{-2}$ by Theorem 1.1, it follows that

$$
\left\|J_{2}\right\|_{1} \leq c \int_{t / 2}^{t}(t-\tau)^{-1 / 2}(1+\tau)^{-2} d \tau \leq c(1+t)^{-3 / 2} \leq c(1+t)^{-1} .
$$

This proves (3.13). Now we apply (3.13) and estimate $\|u\|_{\infty} \leq c(1+t)^{-3 / 2}$ to

$$
u(t)=E_{t / 2} * u(t / 2)-\int_{t / 2}^{t}(\nabla V)(t-\tau) *(u \otimes u)(\tau) d \tau
$$

and find that

$$
\begin{aligned}
\|u(t)\|_{\infty} & \leq c t^{-1}\|u(t / 2)\|_{1}+c \int_{t / 2}^{t}(t-\tau)^{-1 / 2}\|u\|_{\infty}^{2} d \tau \\
& \leq c t^{-2}+c \int_{t / 2}^{t}(t-\tau)^{-1 / 2}(1+\tau)^{-3} d \tau \leq c t^{-2} .
\end{aligned}
$$

Hence $\|u(t)\|_{\infty} \leq c(1+t)^{-2}$. This, together with (3.13), gives

$$
\|u(t)\|_{r} \leq\|u(t)\|_{\infty}^{1-1 / r}\|u(t)\|_{1}^{1 / r} \leq c(1+t)^{-1-(1-1 / r)} \quad(1 \leq r \leq \infty) .
$$


Next, we prove

$$
|u(x, t)| \leq c(1+|x|)^{-4}
$$

Note that

$$
\left|\partial_{x}^{\alpha} E_{t}(x)\right| \leq c_{\alpha}|x|^{-2-|\alpha|}, \quad\left|\partial_{x}^{\alpha} V(x, t)\right| \leq c_{\alpha}|x|^{-2-|\alpha|} \quad(|\alpha| \geq 0) .
$$

We write

$$
I=\left(\int_{|y| \leq|x| / 2}+\int_{|y| \geq|x| / 2}\right) E_{t}(x-y) a(y) d y \equiv I_{1}+I_{2} .
$$

By (3.11), we have

$$
\begin{aligned}
I_{1}= & \int_{|y| \leq|x| / 2} E_{t}^{(2)}(x, y) a(y) d y-E_{t}(x) \int_{|y| \geq|x| / 2} a(y) d y \\
& +\nabla E_{t}(x) \cdot \int_{|y| \geq|x| / 2} y a(y) d y \equiv I_{11}+I_{12}+I_{13} .
\end{aligned}
$$

The definition (3.12) of $E_{t}^{(2)}$, the assumption on $a$ and (3.16) together imply

$$
\begin{aligned}
& \left|I_{11}\right| \leq c|x|^{-4} \int_{|y| \leq|x| / 2}(1+|y|)^{2}|a(y)| d y \leq c|x|^{-4} \int(1+|x|)^{2}|a(y)| d y=c|x|^{-4}, \\
& \left|I_{12}\right| \leq c|x|^{-2} \int_{|y| \geq|x| / 2}|y|^{-2}|y|^{2}|a(y)| d y \leq c|x|^{-4} \int|y|^{2}|a(y)| d y=c|x|^{-4}, \\
& \left|I_{13}\right| \leq c|x|^{-3} \int_{|y| \geq|x| / 2}|y|^{-1}|y|^{2}|a(y)| d y \leq c|x|^{-4} \int|y|^{2}|a(y)| d y=c|x|^{-4} \\
& \left|I_{2}\right| \leq c \int_{|y| \geq|x| / 2} E_{t}(x-y)(1+|y|)^{-4} d y \leq c(1+|x|)^{-4} .
\end{aligned}
$$

Hence $|I| \leq c(1+|x|)^{-4}$. Consider next

$$
J=-\int_{0}^{t}\left(\int_{|y| \leq|x| / 2}+\int_{|y| \geq|x| / 2}\right)(\nabla V)(x-y, t-\tau) \cdot(u \otimes u)(y, \tau) d y d \tau \equiv J_{1}+J_{2} .
$$

By (3.11) we can write $J_{1}$ as

$$
\begin{aligned}
J_{1}= & -\int_{0}^{t} \int_{|y| \leq|x| / 2}\left(\nabla V^{(1)}\right)(x, y, t-\tau) \cdot(u \otimes u)(y, \tau) d y d \tau \\
& +\int_{0}^{t}(\nabla V)(x, t-\tau) \cdot \int_{|y| \geq|x| / 2}(u \otimes u)(y, \tau) d y d \tau \equiv J_{11}+J_{12} .
\end{aligned}
$$


So by (3.10), (3.16) and (3.12) we get

$$
\left|J_{11}\right| \leq c|x|^{-4} \int_{0}^{\infty} \int|y||u(y, \tau)|^{2} d y d \tau \leq c|x|^{-4} .
$$

Moreover, Theorem 1.1 implies $|u(y, \tau)|^{2} \leq c(1+|y|)^{-7 / 2}(1+\tau)^{-5 / 4}$; so (3.16) gives

$$
\begin{aligned}
\left|J_{12}\right| & \leq c|x|^{-3} \int_{0}^{t} \int_{|y| \geq|x| / 2}(1+|y|)^{-7 / 2}(1+\tau)^{-5 / 4} d y d \tau \\
& \leq c|x|^{-4} \int_{0}^{t} \int_{|y| \geq|x| / 2}(1+|y|)^{-5 / 2}(1+\tau)^{-5 / 4} d y d \tau \\
& \leq c|x|^{-4} \int_{0}^{\infty}(1+|y|)^{-5 / 2}(1+\tau)^{-5 / 4} d \tau \leq c|x|^{-4} .
\end{aligned}
$$

Using $|u(y, \tau)|^{2} \leq c(1+|y|)^{-5}(1+\tau)^{-1 / 2}$ which follows from Theorem 1.1, we estimate $J_{2}$ as

$$
\begin{aligned}
\left|J_{2}\right| & \leq \int_{0}^{t} \int_{|y| \geq|x| / 2}|\nabla V(x-y, t-\tau)||u(y, \tau)|^{2} d y d \tau \\
& \leq c \int_{0}^{t} \int_{|y| \geq|x| / 2}|\nabla V(x-y, t-\tau)|(1+|y|)^{-5}(1+\tau)^{-1 / 2} d y d \tau \\
& \leq c(1+|x|)^{-5} \int_{0}^{t}(t-\tau)^{-1 / 2}(1+\tau)^{-1 / 2} d \tau \leq c(1+|x|)^{-5} \leq c(1+|x|)^{-4} .
\end{aligned}
$$

Collecting terms gives (3.15). By (3.14) and (3.15), Theorem 1.3 is valid for $m=1$.

Now, (3.15) and (3.14) with $r=1$ ensure $\int(1+|y|)^{4}|u(y, \tau)|^{2} d y \leq c\|u(\tau)\|_{1}$ $\leq c(1+\tau)^{-1}$; and so, by (3.14) with $r=2$,

$$
\int(1+|y|)^{3}|u|^{2} d y \leq\left(\int(1+|y|)^{4}|u|^{2} d y\right)^{3 / 4}\left(\int|u|^{2} d y\right)^{1 / 4} \leq c(1+\tau)^{-3 / 2}
$$

It follows that

$$
\int_{0}^{\infty} \int(1+|y|)^{3}|u(y, \tau)|^{2} d y d \tau<\infty .
$$

Next, suppose $m=2$; so $a$ is $\mathscr{D}_{4}$-covariant, $(1+|y|)^{5} a \in \boldsymbol{L}^{\infty}\left(\boldsymbol{R}^{2}\right)$ and $(1+|y|)^{3} a \in \boldsymbol{L}^{1}\left(\boldsymbol{R}^{2}\right)$. Then the solution $u$ is $\mathscr{D}_{4}$-covariant. By Proposition 3.2, Corollary 3.3 and (3.17), we have 


$$
\begin{aligned}
u(x, t)= & \int E_{t}^{(3)}(x, y) a(y) d y-\int_{0}^{t / 2} \int\left(\nabla V^{(2)}\right)(x, y, t-\tau) \cdot(u \otimes u)(y, \tau) d y d \tau \\
& -\int_{t / 2}^{t}(\nabla V)(t-\tau) *(u \otimes u)(\tau) d \tau .
\end{aligned}
$$

As in the case $m=1$, we can show that (3.17), (3.18) and assumptions on $a$ together imply

$$
\|u(t)\|_{r} \leq c(1+t)^{-3 / 2-(1-1 / r)} \quad(1 \leq r \leq \infty) .
$$

Due to (3.17)-(3.19), we deduce from Proposition 3.2 and Corollary 3.3 that

$$
|u(x, t)| \leq c(1+|x|)^{-5},
$$

and this proves Theorem 1.3 for $m=2$. (The proof of (3.20) is completely parallel to that of (3.15), so omitted here.) By (3.20) and (3.19) with $r=1$, we obtain

$$
\int_{0}^{\infty} \int(1+|y|)^{5}|u(y, \tau)|^{2} d y d \tau<\infty .
$$

By (3.21), Proposition 3.2 and Corollary 3.3, we can proceed to the proof of the case $m=3$.

Repeating these processes eventually completes the proof of Theorem 1.3 for all $m \in N$. that

Remarks. (i) We know ([16], [18]) the existence of smooth solutions $u$ such

$$
\|u(t)\|_{r} \leq c_{r} e^{-\gamma_{r} t} \quad(1 \leq r \leq \infty, t>0) .
$$

But, nothing is known about the existence of solutions with decay properties that interpolate between the algebraic decay (1.13) and the exponential decay (3.22).

(ii) Define

$$
\boldsymbol{L}^{r}= \begin{cases}\dot{\boldsymbol{B}}_{1,1}^{2(1-1 / r)} & \left(\frac{2}{m+3}<r<1\right), \\ \dot{\boldsymbol{B}}_{1, \infty}^{-(m+1)} & \left(r=\frac{2}{m+3}\right)\end{cases}
$$

where $\dot{B}_{p, q}^{s}=\dot{B}_{p, q}^{s}\left(\boldsymbol{R}^{2}\right)$ is the homogeneous Besov spaces ([1]). Then we can prove

$$
\|u(t)\|_{r} \leq c(1+t)^{-(m+1) / 2-(1-1 / r)} \quad\left(\frac{2}{m+3} \leq r \leq \infty\right)
$$


as in [12], using the techniques given in [4], [14], [15] and [19]. The details are omitted here.

Acknowledgments. Both authors are grateful to the referee for valuable comments. HE is partially supported by the Key Project of the National Natural Science Foundation, No. 10431060; the National Natural Science Foundation, No. 10371125; and the Beijing's Natural Science Foundation, No. 1042003, of People's Republic of China. MiYaKawa is supported in part by Grant-in-Aid for Scientific Research, No. 15340054, from the Japan Society for the Promotion of Science.

\section{References}

[1] Bergh, J. and Löfström, J., Interpolation Spaces, Springer-Verlag, Berlin-New York, 1976.

[2] Brandolese, L., On the localization of symmetric and asymmetric solutions of the NavierStokes equations in $\boldsymbol{R}^{n}, \quad$ C. R. Acad. Sci. Paris, Sér. I Math., 332, No. 2 (2001), 125-130.

[ 3 ] Brandolese, L., Space-time decay of Navier-Stokes flows invariant under rotations, Math. Ann., 329 (2004), 689-706.

[ 4 ] Coifman, R., Lions, P.-L., Meyer, Y. and Semmes, S., Compensated compactness and Hardy spaces, J. Math. Pures et Appl., 72 (1993), 247-286.

[ 5] Fujigaki, Y. and Miyakawa, T., Asymptotic profiles of nonstationary incompressible NavierStokes flows in the whole space, SIAM J. Math. Anal., 33 (2001), 523-544.

[6] Giga, Y., Miyakawa, T. and Osada, H., Two-dimensional Navier-Stokes flow with measures as initial vorticity, Arch. Rational Mech. Anal., 104 (1988), 223-250.

[7] Grove, L. C. and Benson, C. T., Finite Reflection Groups, 2nd ed., Springer-Verlag, New York, 1985.

[ 8 ] He, C. and Miyakawa, T., On $L^{1}$-summability and asymptotic profiles for smooth solutions to Navier-Stokes equations in a 3D exterior domain, Math. Z., 245 (2003), 387-417.

[9] Kajikiya, R. and Miyakawa, T., On $L^{2}$ decay of weak solutions of the Navier-Stokes equations in $\boldsymbol{R}^{n}$, Math. Z., 192 (1986), 135-148.

[10] He, C. and Miyakawa, T., Nonstationary Navier-Stokes flows in a two-dimensional exterior domain with rotational symmetries, Indiana Univ. Math. J., to appear.

[11] Masuda, K., Weak solutions of the Navier-Stokes equations, Tôhoku Math. J., 36 (1984), 623-646.

[12] Miyakawa, T., On upper and lower bounds of rates of decay for nonstationary Navier-Stokes flows in the whole space, Hiroshima Math. J., 32 (2002), 431-462.

[13] Miyakawa, T., Notes on space-time decay properties of nonstationary incompressible NavierStokes flows in $\boldsymbol{R}^{n}$, Funkcial. Ekvac., 45 (2002), 271-289.

[14] Miyakawa, T., Application of Hardy space techniques to the time-decay problem for incompressible Navier-Stokes flows in $\boldsymbol{R}^{n}$, Funkcial. Ekvac., 41 (1998), 383-434.

[15] Miyakawa, T., Hardy spaces of solenoidal vector fields, with applications to the NavierStokes equations, Kyushu J. Math., 50 (1996), 1-64.

[16] Miyakawa, T. and Schonbek, M. E., On optimal decay rates for weak solutions to the Navier-Stokes equations in $\boldsymbol{R}^{n}$, Math. Bohem., 126 (2001), 443-455.

[17] Neumann, P. M., Stoy, G. A. and Thompson, E. C., Groups and Geometry, Oxford University Press, Oxford, 1994. 
[18] Schonbek, M. E., Schonbek, T. and Süli, E., Large time behavior of solutions to the magneto-hydrodynamic equations, Math. Ann., 304 (1996), 717-756.

[19] Stein, E. M., Harmonic Analysis, Princeton University Press, Princeton, NJ, 1993.

[20] Wiegner, M., Decay results for weak solutions of the Navier-Stokes equations in $\boldsymbol{R}^{n}$, J. London Math. Soc., 35 (1987), 303-313.

\author{
nuna adreso: \\ Cheng $\mathrm{He}$ \\ Institute of Applied Mathematics \\ Academy of Mathematics and System Science \\ Academia Sinica \\ Beijing 100080 \\ China \\ E-mail: chenghe@amss.ac.cn \\ Tetsuro Miyakawa \\ Division of Mathematics and Physics \\ Graduate School of Natural Science and \\ Technology \\ Kanazawa University \\ Kanazawa 920-1192 \\ Japan \\ E-mail: miyakawa@kenroku.kanazawa-u.ac.jp
}

(Ricevita la 5-an de januaro, 2005)

(Reviziita la 4-an de marto, 2005) 